\title{
CONSECUENCIAS PEDAGÓgICAS DE LA TEORÍA FICHTEANA DEL DERECHO NATURAL
}

\author{
PEDAGOGICAL CONSEQUENCES OF THE FICHTEAN THEORY OF \\ RIGHT
}

HÉCTOR OSCAR ARRESE IGOR ${ }^{i}$

\begin{abstract}
RESUMEN
Fichte intentó desarrollar una teoría del derecho natural con independencia de la ética, fundado únicamente en su concepción de la subjetividad. Sin embargo, intentaré mostrar que el derecho juega allí una función pedagógica fundamental, dado que es la garantía de la relación de mutuo reconocimiento que hace posible la autoconciencia. El principio del derecho tiene entonces una función pedagógica, en la medida en que permite a cada sujeto tomar conciencia de sus capacidades e iniciar cursos de acción libres. Por lo tanto, el derecho tiene también una dimensión moral, contra el objeto inicial de Fichte de construir una teoría del derecho moralmente neutra.

Palabras clave: Derecho. Subjetividad. Fichte. Moral. Pedagogía.
\end{abstract}

\begin{abstract}
Fichte tried to develop a theory of natural right independently of ethics, based only in his conception of subjectivity. Nevertheless, I will try to show that right plays there a fundamental pedagogical role, because is the guarantee of the relationship of mutual recognition which makes possible the self-conciousness. The principle of right has therefore a pedagogical function, insofar as it allows each subject to be aware of his capabilities and to initiate courses of action. As a result, right has also a moral dimension, against Fichte's initial aim to build a morally neutral theory of right.
\end{abstract}

Keywords: Right. subjectivity. Fichte. Morals. Pedagogy.

El problema de la función pedagógica del derecho tiene larga data y ha recibido diversas respuestas. Entre otras propuestas, puede señalarse la idea hobbesiana de que el derecho a lo sumo puede contener y encauzar los impulsos egoístas y perjudiciales de los hombres (Hobbes, 1651). Por el contrario, Rousseau consideraba que el derecho es una mediación fundamental para hacer posible una convivencia social donde el hombre pueda desarrollar sus mejores tendencias naturales, superando la corrupción a la que lo habían sometido las costumbres de la civilización y la distribución excesivamente asimétrica de la propiedad privada (Rousseau, 1762). Por otro lado, Axel Honneth ha mostrado el rol fundamental que juega el derecho a la hora de construir relaciones de reconocimiento intersubjetivo, que permitan a los sujetos construir una identidad estable y comprenderse a sí mismos como sujetos moralmente responsables (Honneth, 1992, pp. 173-195). 
Suele señalarse el capítulo de la "Autoconciencia" de la Fenomenología del Espíritu, con su célebre dialéctica del amo y el esclavo, como el momento fundacional en cuanto a la teoría del reconocimiento (Hegel, 1807, pp. 127-136). Sin embargo, puede hacerse referencia a otro texto anterior, el Fundamento del derecho natural a partir de los principios de la Doctrina de la Ciencia, publicado por Johann G. Fichte en 1796/1797, en el que encontramos un tratamiento complejo y sistemático de la cuestión del reconocimiento.

Fichte intentó desarrollar una teoría del derecho natural con independencia de la ética, fundado únicamente en su concepción de la subjetividad. Sin embargo, intentaré mostrar que el derecho juega allí una función pedagógica fundamental, dado que es la garantía de la relación de mutuo reconocimiento que hace posible la autoconciencia. El principio del derecho tiene entonces una función pedagógica, en la medida en que permite a cada sujeto tomar conciencia de sus capacidades e iniciar cursos de acción libres. Por lo tanto, el derecho tiene también una dimensión moral, contra el objeto inicial de Fichte de construir una teoría del derecho moralmente neutra.

\section{Autoconciencia y Reconocimiento}

Fichte intenta deducir el derecho natural como condición de la autoconciencia, en un intento por refundar la ciencia jusnaturalista (Renaut, 2001, p. 91). La autoconciencia del yo es su capacidad para atribuirse a sí mismo la capacidad de conocer objetos del mundo sensible. Por lo tanto se trata de una actividad que "vuelve sobre sí misma", es decir que tiene un carácter reflexivo (Fichte, 1796/1797, p. 17). La autoconciencia es la conciencia de que el yo es un agente, no una mera sustancia dada de antemano a la acción. Por lo tanto, en la medida en que querramos entender la subjetividad, la pregunta de cómo es posible la autoconciencia es legítima.

Fichte intenta explicar la autoconciencia a partir de un momento previo a la realización de la acción de conocer un objeto (setzen). Se trata de la acción de proponerse el fin de conocer el objeto (Zwecksetzung). Cuando el yo se propone la acción de conocer, elabora un concepto de su objetivo, en el que él aparece como el autor de dicha acción y el objeto es aquello que él pone o conoce. Si analizamos el concepto del objetivo de la acción, podemos intentar explicar el objeto a partir de la actividad libre del sujeto que lo conoce.

El idealismo de Fichte surge, efectivamente, de una crítica radical del entendimiento humano común (das gemeine Menschenverstand), según el cual los objetos existen 
independientemente de que los conozcamos o no y, por lo tanto, la razón debe adaptarse pasivamente a la índole del objeto a conocer. Fichte sostiene que el entendimiento humano común implica una posición dogmática, porque niega la libertad del sujeto a la hora de conocer el objeto (Fichte, 1796/1797, p. 25).

Por lo tanto, Fichte sostiene que es el yo quien pone el objeto, a través de la actividad libre por medio de la que lo conoce. Esto significa que el objeto puede explicarse gracias a la actividad libre del sujeto. Ahora bien, la autoconciencia no puede explicarse de esta manera, porque el yo es un sujeto finito y necesita siempre tener conciencia de contenidos particulares y determinados. Es decir que no se trata de una conciencia absolutamente libre, sino que el yo debe someter voluntariamente su libertad a las leyes de la naturaleza, para poder conocer un objeto (Fichte, 1796/1797, pp. 18-19). En consecuencia, no puede pensarse una autoconciencia existiendo antes de comenzar a conocer el objeto, por lo que no puede explicarse este último a partir de la primera.

Por esta razón Fichte emprende un segundo camino, consistente en explicar la actividad libre del yo a partir del objeto. Sin embargo, su estrategia se ve abocada al fracaso, porque el conocimiento del objeto es en realidad producto de la actividad del sujeto, como se dijo más arriba. Finalmente, Fichte se ve inmerso en un círculo vicioso, en el que siempre tiene que suponer que la autoconciencia ya está en funcionamiento, que es lo que en realidad debe explicar (Fichte, 1796/1797, p. 30). Luego de haber fracasado en el primer camino emprendido, Fichte decide cambiar el esquema interpretativo sujeto-objeto por un nuevo esquema, que ya no se da en el marco de la intencionalidad sino en el de una relación intersubjetiva (Fichte, 1796/1797, p. 32). Dicho de otro modo, el otro deja de ser un objeto y pasa a ser otro sujeto, un alter ego (Stolzenberg, 1995, p. 80).

Pero no se trata de una relación intersubjetiva en general, sino que en ella el otro sujeto debe transmitirle al yo la idea de que él es un ser racional y que puede llevar a cabo la acción de conocer un objeto en el mundo. Esto es posible gracias a la acción de la exhortación (Aufforderung), en la que el otro invita enérgicamente al yo a actuar por sí mismo (Fichte, 1796/1797, p. 33). Dicho de otro modo, se trata de una invitación a la autodeterminación.

Se trata entonces de una interrelación tal que el yo puede sentirse reconocido como un ser racional por el otro. De este modo, el yo puede acceder a la autoconciencia y atribuirse a sí mismo la capacidad de actuar (Fichte, 1796/1797, p. 34). Esto implica a su vez que la exhortación a la acción no puede tener cualquier forma, sino que debe cumplir con ciertos requisitos para poder lograr el objetivo de reconocer al yo como una autoconciencia. Ante 
todo, el otro no debe coaccionar al yo de ningún modo para que responda de tal o cual manera a su exhortación, sino más bien dejarlo libre para que decida o no actuar (Crone, 2005, p. 142). Si el otro coaccionara al yo, lo estaría tratando como a un objeto y no como a un sujeto, y no le estaría transmitiendo la idea de que es un ser libre y racional.

De allí la necesidad de que el otro le abra al yo una esfera de acciones libres, entre las cuales pueda elegir una. El otro debe respetar la esfera de acciones del yo, quien a su vez debe permitirle al otro un conjunto igual de acciones alternativas. Esta determinación mutua de esferas de acción es el contenido del principio del derecho (Rechtssatz) (Fichte, 1796/1797, p. 52).

El derecho es entonces un presupuesto pragmático de la acción misma de la exhortación $y$, en tanto que tal, de la relación mutua de reconocimiento. Dado que la relación de reconocimiento tiene la función pedagógica de despertar al yo a su autoconciencia, el derecho también tiene la misma función. De este modo, el derecho no puede ser pensado independientemente de la ética, dado que la relación legal tiene como motivación el respeto por la libertad del otro en tanto que fin en sí mismo (Wood, 1990, p. 83).

Como señala el mismo Fichte, el hombre ser convierte en tal sólo cuando está entre otros seres humanos (Fichte, 1796/1797, p. 39). Sin embargo, el principio del derecho permanece todavía como un enunciado abstracto, porque falta aún deducir la constitución del individuo particular que actuará en cada caso concreto. Pero para ello es necesario previamente deducir el cuerpo propio del yo.

Fichte argumenta que el yo puede saber que dispone de una esfera de acciones libres abierta por el otro en la medida en que puede poner ese conjunto de acciones como existiendo en el mundo, independientemente de su actividad de ponerlo. En caso contrario, se trataría de una mera ilusión o de una afirmación arbitraria pero sin contenido real. La esfera de acciones puesta por el yo en el mundo sensible es el cuerpo (Leib), que no es lo mismo que el mero organismo biológico (Körper) (Fichte, 1796/1797, p. 59).

La diferencia radica en que, mientras que el organismo es una totalidad estructurada que tiene como última función la autoconservación, el cuerpo es un conjunto de alternativas de acción que el yo se atribuye a sí mismo, porque el otro se la ha reconocido previamente. Es decir que el cuerpo es en realidad una construcción jurídica, producto de la relación de reconocimiento recíproco que hace posible la autoconciencia. Por lo tanto, el principio del derecho le enseña al yo que él tiene un cuerpo y que puede realizar acciones en el mundo sensible gracias a esta mediación fundamental. 
De este modo se completa la deducción de la autoconciencia a partir del reconocimiento del otro y ha quedado claro el rol derecho pedagógico del derecho, como garantía de dicha relación. De lo argumentado hasta aquí puede concluirse que el yo no puede convertirse en un ser humano pleno en medio de una forma de gobierno donde su esfera de acción sea invadida arbitrariamente por el Estado. Esto nos lleva a su vez a la cuestión de si el derecho aplicado tiene en Fichte la misma función pedagógica que el derecho puro.

\section{EI derecho como mediación estratégica e instrumental}

La relación de mutuo reconocimiento que hace posible la autoconciencia exige que cada miembro quede libre para responder o no a la exhortación del otro, para evitar la coacción y la cosificación consecuente. Sin embargo, esto acarrea el problema de que el principio del derecho no está garantizado, dado que queda abierta la posibilidad de que alguno de los miembros atenten contra él. Por lo tanto, la teoría fichteana necesita de un reaseguro para garantizar el principio del derecho. Se trata necesariamente del Estado, única mediación que puede monopolizar la fuerza para asegurar la propiedad y la libertad de todos (Fichte, 1796/1797, p. 150).

Pero el Estado debe ser diseñado de modo tal de proteger a los ciudadanos contra la eventualidad de que alguno de ellos deje de respetar el principio del derecho, para lo que debe suponer que cada ciudadano se comportará de modo egoísta, aunque de hecho eso no ocurra (Fichte, 1796/1797, pp. 142 y 273). Siguiendo las huellas de Hobbes, Fichte sostiene que los ciudadanos deben entregar toda su libertad al Estado para que pueda imponer la ley y evitar las transgresiones (Fichte, 1796/1797, p. 161; Oncina Coves, 2004, p. 214).

En consecuencia, la relación legal pierde en el derecho aplicado la fuerza pedagógica que tiene en el derecho puro, porque cada sujeto se incorpora a la vida estatal únicamente para proteger su auto-interés. Es decir que no se espera que los ciudadanos modifiquen sus preferencias como resultado de su participación en la vida pública, sino que a lo sumo negocien sus derechos y prerrogativas para proteger su libertad del mejor modo posible. Ante todo, el Estado permitirá a cada ciudadano conservarse a sí mismo en la existencia biológica (Fichte, 1796/1797, p. 212).

Por lo tanto, existe un quiebre importante desde el punto de vista pedagógico entre las funciones que tiene el derecho en el ámbito de la fundamentación de la teoría y aquél que le corresponde al ámbito de la aplicación. Como señaló el mismo Hegel, en el derecho fichteano 
los ciudadanos no pueden autogobernarse, porque no aprenden a hacerlo como consecuencia de su vida cívica, en la medida en que delegan sus prerrogativas en un tercero (Hegel, 1802/1803, p. 474).

\section{Conclusiones}

La teoría fichteana del derecho natural es un momento fundacional de las teorías sobre el reconocimiento, pero además nos permite reflexionar sobre la función antropológica que tiene el derecho, más allá de lo político y lo judicial. Es decir que el derecho juega un rol fundamental en la constitución de la subjetividad, en la medida en que estructura la relación de reconocimiento que permite al yo tomar conciencia de sus posibilidades de acción.

El idealismo de Fichte tiene una carga metafísica importante, en cuanto que entiende al yo como la autoconciencia que pone el mundo. Desde este punto de vista, el mundo no existe independientemente de la actividad cognoscitiva del yo, pero el yo debe actuar como si efectivamente ocurriera lo contrario. Para no caer en el relativismo, el yo necesariamente debe someter su libertad a las leyes de la naturaleza, como si no dependieran de la espontaneidad absoluta del yo.

Sin embargo, a pesar de que no estamos seguros de si el mundo existe, al menos tal como lo conocemos, no podemos dudar de que existen los demás, dado que son la condición de posibilidad de nuestra misma autoconciencia. Dicho de otro modo, los otros están dentro de nosotros, es decir que son un momento constitutivo de nuestra propia mismidad.

El derecho se ubica en esta encrucijada en la que se juega la existencia del yo, en la medida en que es reconocido o cosificado por el otro. Lamentablemente el derecho no conserva este rol en el ámbito del derecho aplicado, esto es en la teoría del Estado. A diferencia de la teoría de Honneth, la concepción fichteana deja de lado el rol pedagógico que el derecho debe jugar en la vida pública de nuestras comunidades. 


\section{Notas:} ${ }^{1}$ Héctor Oscar Arrese Igor es Investigador Adjunto de la Comisión Nacional de Investigaciones Científicas y
Técnicas (CONICET) y Prof. Asociado Ordinario de Filosofía de los Siglos XIX y XX en la Universidad
Pedagógica Provincial (Argentina). 


\section{Referencia Bibliografía}

Crone, K., (2005), Fichtes Theorie konkreter Subjektivität. Untersuchungen zur "Wissenschaftslehre nova methodo", Gotinga: Vandenhoeck \& Ruprecht.

Fichte, J. G., (1796/1797), Grundlage des Naturrechts nach Principien der Wissenschaftslehre, reimpreso en: (1971), Johann Gottlieb Fichte: Sämmtliche Werke. Hrsg. v. Immanuel Hermann Fichte. Berlín: Walter de Gruyter, Band III.

Hobbes, Th., (1651), Leviathan, reimpreso en: (1998), Oxford: Oxford University Press.

Hegel, G. W. F., (1802/1803), "Über die wissenschaftlichen Behandlungsarten des Naturrechts, seine Stelle in der praktischen Philosophie und sein Verhältnis zu den positiven Rechtswissenschaften", en Kritisches Journal der Philosophie, Bd. II, Stück 2, [November/Dezember] 1802, und Stück 3, [Mai/Juni] 1803. Citado según la paginación de la edición: Hegel, G. W. F., (1970), Werke in 20 Bänden, Frankfurt: Suhrkamp Verlag, Band 2. Hegel, G. W. F., (1807), Phänomenologie des Geistes, citado según la paginación de: (1988), Hamburg: Meiner Verlag.

Honneth, A., (1992), Kampf um Anerkennung. Zur moralischen Grammatik sozialer Konflikte, Frankfurt: Suhrkamp.

Oncina Coves, F., (2004), "Fichtes Kritik des aufklärerischen Republikanismus", en: De Pascale, C., Fuchs, E., Ivaldo, M., Zöller, G., (hrsg.), (2004), Fichte und die Aufklärung, Hildesheim-Zürich-New York: Georg Olms Verlag, pp. 213-226.

Renaut, A., (2001), "Deduktion des Rechts (Dritter Lehrsatz: § 4), en: Merle, J. Ch., (Hrsg.), Johann Gottlieb Fichte. Grundlage des Naturrechts, Berlín: Klassiker Auslegen, Band 24, Akademie Verlag, pp. 81-95.

Rousseau, J.-J., (1762), Du Contrat Social, citado según la paginación de: (1964), JeanJacques Rousseau. Oeuvres Complètes, Vol. III, París, Éditions Gallimard, pp. 281-470.

Stolzenberg, J., (1995), "Fichtes Begriff des praktischen Selbstbewusstseins", en: Hogrebe, W., (1995), Fichtes Wissenschaftslehre. 1794. Philosophische Resonanzen, Frankfurt am Main, Suhrkamp, pp. 71-95.

Wood, A., (1990), Hegel's Ethical Thought, Cambridge, New York, Port Chester, Melbourne, Sidney, Cambridge University Press. 